\title{
E-conversation with Professor Mahmoud El-Halwagi
}

\author{
Subhas K. Sikdar
}

Published online: 13 May 2014

(c) Springer-Verlag Berlin Heidelberg (outside the USA) 2014

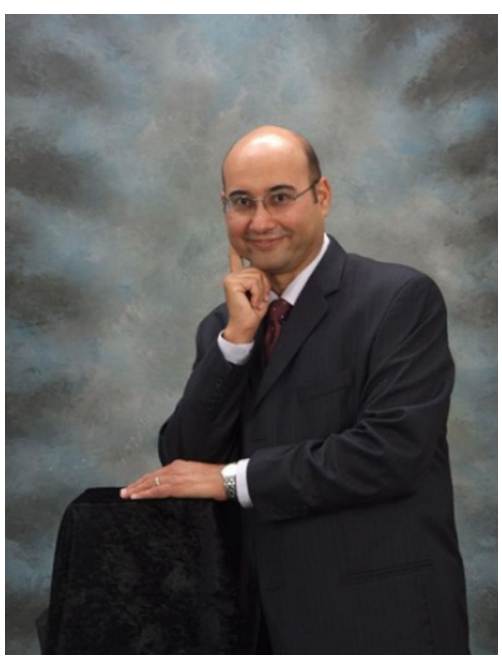

Mahmoud El-Halwagi (MEH) is Professor and Holder of the McFerrin Professorship at the Artie McFerrin Department of Chemical Engineering at Texas A\&M University. His main area of research is sustainable design through process integration. He has served as a consultant to numerous companies. Dr. El-Halwagi holds a $\mathrm{PhD}$ from the University of California, Los Angeles (UCLA), and an MS and a BS from Cairo University, all in chemical engineering. For more biographical information, the reader is referred to http://engineering.tamu.edu/chemical/people/ melhalwagi.

(SKS): Your graduate work on the mass exchange networking idea at UCLA was a path-breaking technical work. I view this development as a logical advancement over the heat exchange networking which had already achieved

S. K. Sikdar ( $\square)$

U.S. Environmental Protection Agency, Cincinnati, OH, USA

e-mail: sikdar.subhas@epa.gov commercial applications especially in petroleum refining industry. You have successfully promoted process integration that incorporates both of these ideas in minimizing energy and material usage in process systems design and re-design and have made the important linkage to process sustainability. Sustainability is looked upon as a systems analysis issue in engineering discipline, and process integration is also that. But process integration is also being researched worldwide resulting in newer and newer tools. Can you explain for the benefit of the readers if and how what you have been doing is a different approach to process integration?

(MEH): Thank you very much for your kind characterization of my work. By the time I started my $\mathrm{PhD}$ work in the late 1980s, the field of process integration had already made substantial progress in the area of energy integration using systems thinking and tools. My interest was in the area of sustainability which, as you mentioned, involves systems analysis. Instead of confining the research to endof-pipe treatment with focus on individual units and streams, I was convinced that (1) environmental problems for the process industries are best tackled by focusing on the root causes of the problems and not just the symptoms, (2) environmental objectives must be coordinated, and in some cases reconciled, with other process objectives (economic, technical, safety, social, etc.), (3) a "big-picture" approach would yield insights and innovations unseen by the conventional unit-operations approach, and (4) the large number of design alternatives calls for the development of systematic and generally applicable tools. Three decades later, the same principles continue to drive my research.

(SKS): Currently many researchers around the world are following your lead in using the basic principles of mass and energy pinch analyses in process system integration. 
Others are also pursuing process integration for the same overall multi-criteria optimization objectives using other algorithmic techniques. Can you please characterize the various approaches pointing out the differences in approaches and the differences in outcomes of the various techniques? Do the various techniques arrive at the same technological outcomes?

(MEH): The first step in creating a successful process integration tool is to develop a conceptual approach or a "game plan" to systematically solve a general class of problems. This is a difficult task because it involves a combination of creativity and fundamental rigor. Once this conceptual approach is developed, then the implementation and solution can be carried out using different techniques. These include graphical, algebraic, and mathematical programing approaches. Graphical approaches (such as the pinch diagrams for energy, mass, and property integration) are useful in setting top-level performance targets and in visualizing the system from an insightful perspective. Algebraic tools use spreadsheets and may, therefore, be quickly learned and used by process engineers who can integrate them with other spreadsheet tools that they already use. Mathematical programing (such as linear programing, nonlinear programing, mixed integration linear programing, and mixed integer nonlinear programing) is very powerful in optimizing systems while incorporating various constraints and (as you have mentioned) multiple criteria for objectives. When modeled rigorously and solved globally, these mathematical programs provide remarkable results to complex problems. I personally like all three approaches and have developed and used them in various research activities and industrial projects with the choice depending on the available data, the required level of details, and the overall objectives.

(SKS): Thanks for explaining these differences. Mass integration techniques as a logical next step are easier to understand, conceptually. Indeed, mass flows in processes are always associated with sensible and/or latent energy; hence, mass integration subsumes energy integration as well. But property integration is a different objective. Please explain what one would want to achieve by property integration, and what new vistas in design objectives can be opened by this technique.

(MEH): Mass integration is based on tracking different chemicals, identifying performance benchmarks for those chemicals, and developing strategies for the allocation, transformation, and separation of those chemicals. In some cases, the design objectives are not "chemo-centric". Instead, properties and functionalities may be the primary characteristics for design objectives and constraints. For instance, the use of scrubbing solvents depends on their properties such as absorption coefficients and vapor pressure. The design of vapor-liquid equilibrium units depends primarily on relative volatility. The characterization of numerous products is based largely on their properties and not necessarily chemical constituents. Even the impact on the environment may be best assessed through properties that waste discharges impart (e.g., pH, toxicity, and color). There are also some cases that a feedstock may contain numerous compounds (e.g., crude oil, biomass) that are difficult to track and optimize through chemical-based mass integration. In such cases, the design is based on tracking, transforming, allocating, and optimizing properties throughout the process. This is the essence of property integration. In addition to the use of property integration in process design and integration, it also provides a natural interface for the simultaneous process and molecular design when both problems are posed in terms of common properties.

(SKS): Alright, so when looking at property integration, you are essentially trying to match the desired properties of the undesirable chemicals with the same desirable properties with benign chemicals. Is that about right? In this case then you are trying to find chemical species that are already commercially available, or is it also a chemical design challenge? Our Heriberto Cabezas developed PARIS II software to achieve similar ends. Would PARIS II be called a property integration tool? In the latter, however, frequently a mixture would be needed to achieve the objective of property matching and passing a toxicity threshold.

(MEH): The example and tool you mentioned are part of property integration with focus on the molecular selection and/or design aspects. But property integration is much broader because it also accounts for process design. Here are some examples to clarify:

(a) Consider several process units that use external fresh resources because they offer specific properties. Suppose that there are also several process streams and waste streams that may be recycled to reduce the use of the external fresh resources while still meeting the required properties required by the units. This is the direct recycle problem of process integration, and it involves determining how to reroute the recyclable streams (or portions of these streams), mix them and reduce the usage of the fresh streams. The solution must consider all the recyclable streams, fresh sources, process units, and desired property constraints.

(b) Let us take the previous example a step further. If we would like to reduce the usage of the fresh stream below the target that can be achieved by directly recycling the process and waste streams, how should the properties of the recyclable streams be altered and to what extent? This is the recycle problem of property integration with interception (units that can alter the properties of the recyclable streams). 
(c) Another example is when there are several waste streams to be discharged to the environment, and it is required to alter certain characteristics of the waste streams (e.g., BOD, toxicity, color, $\mathrm{COD}$, and $\mathrm{pH}$ ). Instead of designing end-of-pipe treatment units, what changes can we make throughout the process to reach the desired characteristics? These changes include manipulating design and operating variables of the units throughout the process, mixing and rerouting streams, substituting some fresh resources, and adding in-process units to adjust the properties of certain streams. These are all property integration strategies.

(SKS): Because of these process integration techniques, our readers who are not practitioners would be benefited by the knowledge of the level of success that has been achieved using these techniques industrially for process design and operation, for saving material and energy, and generally for making them more efficient. It would be good if you provide a sense of the extent of industrial adoption, either from your own research or from others'.

(MEH): Process integration had its first major success in theory and applications in the area of energy integration in the 1970s and early 1980s. Mass integration was introduced in the late 1980s and property integration was introduced in the early 2000s. Because of this progress, the majority of the earlier industrial applications and adoptions were in the area of energy integration. These were followed by applications via mass integration then property integration. There are hundreds of very successful process integration projects around the world. Some of the results have been reported in review articles and books in raw or "sanitized" forms. The outcome of these projects clearly shows remarkable improvements over "conventional" engineering approaches with substantial savings in the usage of energy, raw materials, and materials utilities, enhancement of yield, and reduction in waste discharge. In addition to these substantial improvements, a key advantage is that process integration empowers the process engineer to methodically identify root causes of the design and operating problems, calculate performance benchmarks, determine implementation strategies, trade off objectives, gain very clear insights, and smoothly navigate through the complexity of the industrial processes.

(SKS): Yes, what you say here is true. I wonder what assessment you have been able to gather on the impact of all these highly trained graduates are having in applying this knowledge in a priori design of plants in chemical or allied industries. I understand that industry adoption of process integration is easier at the retrofit level. Any light you can shed based on your reconnaissance?

(MEH): Indeed, most of the initial applications of process integration were at the retrofitting level. This has gradually changed especially with the introduction of process integration tools into the toolkits of engineering and simulation firms. Additionally, the incorporation of these tools into the undergraduate chemical engineering curriculum has equipped graduates with valuable knowledge and skill set in the area of process integration. This is an important step in technology diffusion. At present, I believe that most grass-root designs include elements of process integration. The more recent advances in process integration will take some time to diffuse into industrial practice but the basic tools have already been widely adopted.

(SKS): In process optimization, from an industrial perspective, especially among competitive processes for similar products, we are primarily obligated to minimize manufacturing costs, while at the same time reduce resource use (materials, water, and energy) as much as possible. This is like saying, the optimized option does not offer minima for all important objectives independently but overall that is the best option under the proviso that cost has to be minimized subject to some business requirements of such objectives as product purity or mandated effluent and emission criteria. Stated this way, we can claim this practice of optimization by process integration is offering relative sustainability. Are we at a stage now that this relative sustainability can be achieved by the mathematical tools at hand? Are there some examples of such accomplishments?

(MEH): Thank you for raising this important issue. Each company has a number of primary objectives (e.g., cost, quality, capital productivity, yield, energy conservation, environmental impact, safety, flexibility, and operability). These objectives may need to be reconciled. Toward this goal, multi-objective optimization is an ideal tool, and it has served in various industrial projects to generate "Pareto curves" that establish tradeoffs among the different objectives. When it comes to the specific objectives of cost and conservation of natural resources (e.g., mass and energy), it is worth noting that when mass and energy integration approaches are properly used, the usage of mass and energy is first reduced while enhancing the profitability of the process. Then, a point of diminishing return is reached after which the company has to decide the extent of additional mass and energy conservation. Such an extent varies from one company to another, from one period to another, and from one society to another.

(SKS): I have one last question for you. You hail from Egypt. Over the years you have traveled widely across the globe to share your expertise with various professionals. You have special relationship with many countries in the Middle East, and Latin and South America. I am curious to know if you can provide a bird's eye view of the 
contributions being made in mathematical approaches to process analysis in the countries that you visit most often.

(MEH): I appreciate the question because it gives me an opportunity to acknowledge my country of origin and to thank my research partners around the world whose contributions have made a significant impact on my career. I owe them a great deal of gratitude. It has been a very rewarding experience to work with my graduate students who came from all over the world. I have also been fortunate to collaborate with professors from various countries. At present, I have active collaboration with professors from different countries especially Canada, China, Colombia, Egypt, Greece, Malaysia, Mexico, Qatar, Saudi Arabia, and South Africa. The research institutions in these countries are showing an increasing level of attention to sustainable development and sustainable design. Some of the topics (e.g., resource conservation, profitability enhancement, and water management) are common in the different countries. Nonetheless, the research in each country may also take a certain flavor reflecting the domestic needs and resources. For instance, key contributions in the optimization-based approaches for integrated biorefineries are coming from Mexico and Colombia. On the other hand, a focal area of research in Qatar and Saudi Arabia is sustainable design of oil and gas processing facilities. There are web sources that provide links to research groups in process systems engineering. For instance, the following web site provides very good information on the process-systems engineering groups in Pan American countries: http://cepac.cheme.cmu.edu/ country.htm.

(SKS): Mahmoud, I thank you very much for sharing your thoughts for the benefit of our readers.

(MEH): Thanks a lot, Subhas. I am very grateful for the opportunity to converse with you and to share my thoughts with the readers of Clean Technologies and Environmental Policy. 